\title{
Terminal holographic complexity
}

\section{J.L.F. Barbón and J. Martín-García}

Instituto de Física Teórica, Universidad Autónoma de Madrid, IFT-UAM/CSIC, C/ Nicolás Cabrera 13, 28049 Madrid, Spain

E-mail: jose.barbon@uam.es, javier.martingarcia1@gmail.com

ABSTRACT: We introduce a quasilocal version of holographic complexity adapted to 'terminal states' such as spacelike singularities. We use a modification of the action-complexity ansatz, restricted to the past domain of dependence of the terminal set, and study a number of examples whose symmetry permits explicit evaluation, to conclude that this quantity enjoys monotonicity properties after the addition of appropriate counterterms. A notion of 'complexity density' can be defined for singularities by a coarse-graining procedure. This definition assigns finite complexity density to black hole singularities but vanishing complexity density to either generic FRW singularities or chaotic BKL singularities. We comment on the similarities and differences with Penrose's Weyl curvature criterion.

KEYwords: Spacetime Singularities, AdS-CFT Correspondence, Black Holes, Gaugegravity correspondence

ArXiv ePrint: 1805.05291 


\section{Contents}

1 Introduction $\quad 1$

2 A quasilocal AC ansatz for terminals 2

$\begin{array}{lll}3 & \text { The local component of the terminal complexity } & 7\end{array}$

3.1 Evanescent terminal complexity 8

$\begin{array}{lll}3.2 & \text { Local terminal complexity and coarse-graining } & 10\end{array}$

4 Terminal monotonicity $\quad 14$

5 Conclusions and outlook $\quad 22$

\section{Introduction}

Spacetime singularities are perhaps the most radical boundary of knowledge in theoretical physics, a condition greatly amplified by the high degree of self-consistency of General Relativity. A number of timelike singularities have been successfully resolved in string theory, through the expedient of exhibiting extra light degrees of freedom localized at the singular locus. Spacelike singularities, on the other hand, are usually regarded as intrinsically associated with strong gravitational dynamics and thus beyond the realm of string perturbation theory.

Very broadly, there are two traditions regarding the interpretation of spacelike singularities: either they must be 'resolved' so as to restore some type of evolution across the singularity, or they must be accepted as true 'spacetime terminals'. To the extent that the black hole singularity is a general guide, the second option is preferred in modern discussions based on holography. On the other hand, the straightforward application of holographic ideas requires an identification of appropriate AdS/CFT boundaries or at least some notion of holographic screen.

Recently, key roles for quantum entanglement and quantum complexity in the workings of holography have been increasingly appreciated. Roughly, the degree of connectivity of space is related to the entanglement of the holographic degrees of freedom, and its volume behaves as a measure of their quantum complexity. In this note we seek to associate holographic measures of quantum complexity to states which are linked to spacelike singularities by time evolution. This program was initiated in previous work $[1,2]$ by the analysis of certain cosmological singularities with controlled AdS/CFT embedding. Here we seek to provide quasilocal notions of complexity which may be abstracted from particular AdS/CFT constructions, and therefore having a larger degree of generality. While we use the volume-complexity (VC) proposal [3] as a heuristic guide, most of our discussion is tailored to the more covariant action-complexity $(\mathrm{AC})$ proposal $[4,5]$. 
The connection between spacetime singularities and complexity has a long history, going back to the occurrence of classical chaos in generic cosmological singularities [6-9] (see [10] for a recent review). In [11] Penrose gave a local criterion for the complexity of a singularity. The basic observation is that 'ordered' singularities, such as those arising in FRW models, have vanishing Weyl curvature, whereas more generic ones, such as those arising in gravitational collapse, have a generically divergent Weyl tensor. Penrose argued that the Weyl criterion would be associated to a large gravitational entropy flowing into the singularity, a suggestion based on the heuristic picture of a generic cosmological crunch, full of chaotic black hole collisions. Since black holes are known to carry entropy, a corresponding notion of entropy may be assigned to the union of all singularities enclosed by the colliding black holes.

One basic observation of this paper is that a suitable version of holographic complexity, rather than entropy, provides a more natural measure of complexity of a singularity. The proposal uses a restriction of the standard $\mathrm{AC}$ ansatz of $[4,5]$ to the causal domain of dependence of the singularity. Furthermore, we will see that a local notion of complexity, different from Penrose's Weyl curvature, can be naturally introduced in the holographic formalism through a coarse-graining procedure.

The paper is organized as follows. In section 2 we lay down some general definitions of 'terminal holographic complexity'. In section 3 we discuss the local contribution to complexity and the coarse-graining procedure. In section 4 we investigate the monotonicity properties of this quantity in some examples of singularities admitting a completely analytic treatment. Finally, in section 5 we offer some conclusions and outlook for plausible generalizations.

\section{A quasilocal AC ansatz for terminals}

A rather intuitive notion of holographic complexity is provided by the $\mathrm{VC}$ ansatz of [3]. Formally, it looks like a generalization of the HRT construction for holographic entanglement entropy $[12,13]$, removing one unit of codimension. One considers extremal codimensionone surfaces anchored on boundary holographic data, and their volume, in an appropriate normalization, yields the VC complexity of the dual state (see also [14]). A more covariant prescription (AC complexity) was subsequently introduced in $[4,5]$, where one is instructed to integrate the bulk classical action over the full causal domain of dependence of the extremal surfaces, henceforth referred as the Wheeler-de Witt (WdW) patch.

The basic physical guide is that either ansatz provides a linear growth of quantum complexity for a high-temperature CFT thermofield double or, in the dual picture, a large eternal AdS black hole,

$$
\frac{d C}{d t} \sim T S \sim M
$$

where $S, T, M$ denote entropy, temperature and mass, respectively. This law is supposed to apply for $t \gg T^{-1}$, up to $O(1)$ coefficients. The detailed dependence on these coefficients is argued to be more uniform for the AC prescription, although the physics is qualitatively 

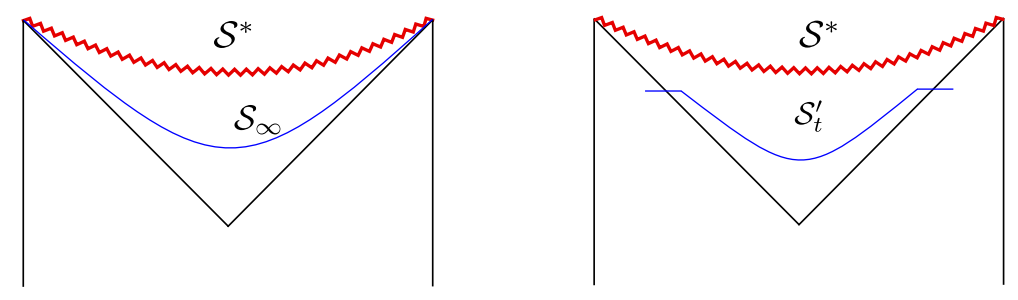

Figure 1. On the left, the codimension-one asymptotic surface $\mathcal{S}_{\infty}$, accounting for the total complexity 'flowing' into the black-hole singularity $\mathcal{S}^{*}$. On the right, the subtracted codimensionone surface $\mathcal{S}_{t}^{\prime}$ which accounts for the time-dependence of VC complexity in the eternal black hole geometry.

the same, at least for large enough temperatures. ${ }^{1}$ Still, some qualitative differences in the AC/VC dichotomy persist, particularly for cold systems, such as near-extremal black holes or cold hyperbolic black holes. This is testimony of our still quite poor understanding of the duality [18-20].

In the benchmark model provided by the eternal black hole spacetime, the central object of interest for the $\mathrm{VC}$ ansatz is the extremal codimension-one surface $\mathcal{S}_{\infty}$ shown in figure 1. This surface maximizes the volume locally and it lies entirely within the black hole interior, i.e. the past causal domain of the singularity.

The growth of complexity within the VC ansatz can be seen as the result of gradually accessing an increasing portion of $\mathcal{S}_{\infty}$. More precisely, the portion of the extremal surface $\mathcal{S}_{t}$ which has a significant contribution to time dependence can be analyzed approximately as composed of two parts: a subset of $\mathcal{S}_{\infty}$ with volume proportional to $t$, and a transition surface at the horizon, whose contribution to the complexity is of order $S$, the entropy of the black hole. Let us denote by $\mathcal{S}_{t}^{\prime}$ this, loosely defined, 'subtracted' surface as indicated in figure 1.

Once we decide to focus on $\mathcal{S}_{t}^{\prime}$ and its asymptotic limit $\mathcal{S}_{\infty}$, we may consider versions of these quantities for any terminal set $\mathcal{S}^{*}$ (which may in particular be a proper subset of a wider one). The reason is that the analog of $\mathcal{S}_{\infty}$ always exists given any spacelike terminal set $\mathcal{S}^{*}$ and its associated past domain of dependence $D^{-}\left(\mathcal{S}^{*}\right)$ (see figure 2). Since the volume is positive and the past boundary of $D^{-}\left(\mathcal{S}^{*}\right)$ is null, the extremal surface is either a local maximum of volume or it coincides with $\mathcal{S}^{*}$ in a degenerate case. The first situation occurs when $\mathcal{S}^{*}$ is a standard singularity of the kind we encounter at black holes and cosmological crunches in General Relativity, since the volume of spatial slices vanishes at such singularities.

A more covariant version of $\mathcal{S}_{\infty}$ and $\mathcal{S}_{t}^{\prime}$ could be obtained by adapting the AC ansatz to this situation (cf. figure 3). Since $\mathcal{S}_{\infty}$ is the extremal surface on $D^{-}\left(\mathcal{S}^{*}\right)$, the natural $\mathrm{AC}$ version of the full terminal complexity of the set $\mathcal{S}^{*}$ is the on-shell action

$$
C\left[\mathcal{S}^{*}\right] \propto I\left[D^{-}\left(\mathcal{S}^{*}\right)\right],
$$

evaluated over the set $D^{-}\left(\mathcal{S}^{*}\right)$. Since this definition only makes reference to the terminal set $\mathcal{S}^{*}$ we regard this notion of complexity as 'quasilocal' and will often denote it as such.

\footnotetext{
${ }^{1}$ It has been conjectured that (2.1) should saturate the Lloyd bound [15]. See, however [16, 17].
} 

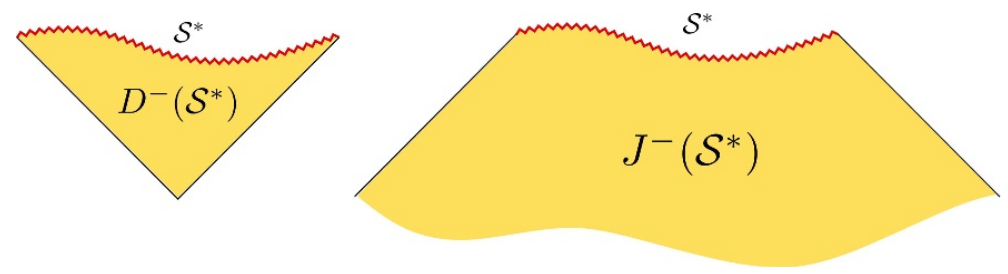

Figure 2. Generic terminal set $\mathcal{S}^{*}$ (in red) and its past domain of dependence $D^{-}\left(\mathcal{S}^{*}\right)$ and causal past $J^{-}\left(\mathcal{S}^{*}\right)$.

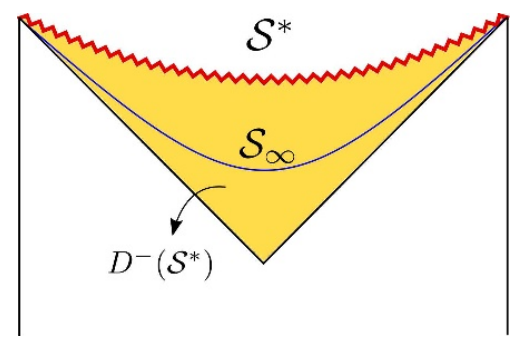

Figure 3. The total VC complexity flowing into the singular set $\mathcal{S}^{*}$ is the volume of the asymptotic surface $\mathcal{S}_{\infty}$. Its $\mathrm{AC}$ analog is the on-shell action integrated over the past domain of dependence $D^{-}\left(\mathcal{S}^{*}\right)$.

Next, a notion of 'time-dependence' can be defined by considering a WdW patch anchored roughly at the exit points of the cut-off surface $\mathcal{S}_{t}^{\prime}$, as indicated in figure 4. A more invariant definition can be obtained by noticing that these WdW patches are nested into one another as time increases. For sufficiently 'late' WdW patches, this 'nesting' extends to the intersections of the WdW patches with the singular set. This suggests that we may use the nested singular subsets as a starting point in the definition of the WdW nested family. To be more precise, let us pick a sequence of terminal subsets $\mathcal{S}_{u}^{*}$, ordered by inclusion in the sense that

$$
\mathcal{S}_{u}^{*} \subset \mathcal{S}_{u^{\prime}}^{*}, \text { for } u<u^{\prime},
$$

and converging to the full terminal set $\mathcal{S}^{*}$ as $u \rightarrow u_{*}$, we can consider a set of WdW patches $\mathcal{W}_{u}$, defined as the intersection between $D^{-}\left(\mathcal{S}^{*}\right)$ and the causal past of $\mathcal{S}_{u}^{*}$,

$$
\mathcal{W}_{u}=J^{-}\left(\mathcal{S}_{u}^{*}\right) \cap D^{-}\left(\mathcal{S}^{*}\right)
$$

For any given $\mathcal{W}_{u}$, its Cauchy surfaces $\Sigma_{u}$ have a common codimension-two boundary $\mathcal{V}_{u}=\partial \Sigma_{u}$ (cf. figure 5), which would hold the 'holographic data' for $\mathcal{W}_{u}$. For example, $\mathcal{V}_{u}$ is a spatial section of the event horizon when $\mathcal{S}^{*}$ is a black-hole singularity. Therefore, we would like to interpret the 'area' of $\mathcal{V}_{u}$ in Planck units ${ }^{2}$ as a measure of the effective number of holographic degrees of freedom 'flowing' into the terminal subset $\mathcal{S}_{u}^{*}$.

In defining the $\mathrm{WdW}$ patches $\mathcal{W}_{u}$ we may give privilege to the 'anchors', namely the codimension-two sets $\mathcal{V}_{u}$, or alternatively we may consider the nested family $\mathcal{S}_{u}^{*}$, as more

\footnotetext{
${ }^{2}$ We henceforth refer to codimension-two volumes as 'areas'.
} 


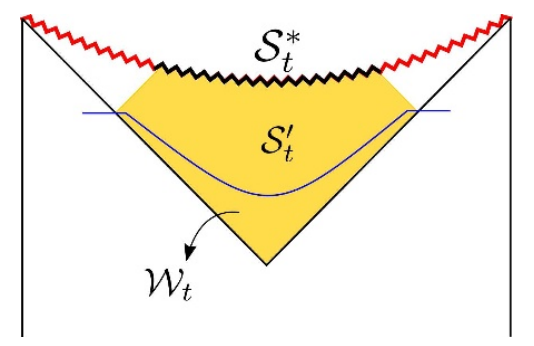

Figure 4. The WdW patch $\mathcal{W}_{t}$, associated to the cut-off surface $\mathcal{S}_{t}^{\prime}$, intersects the singularity at $\mathcal{S}_{t}^{*}$.

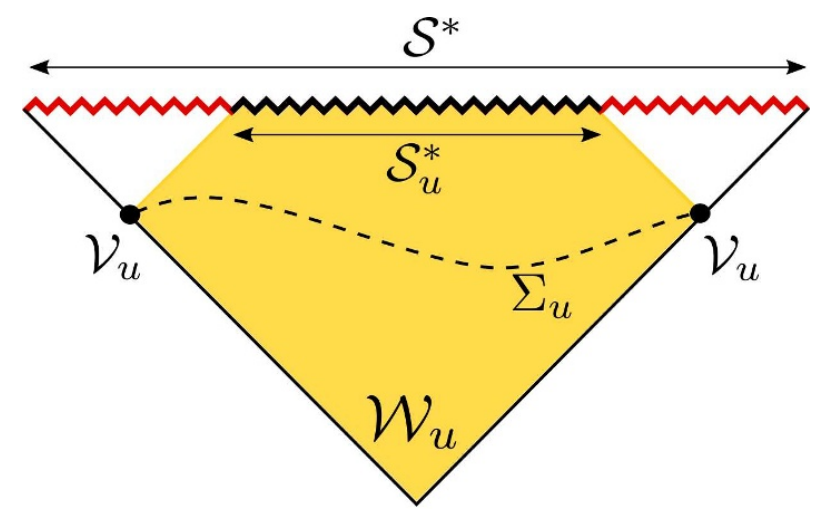

Figure 5. The WdW patch $\mathcal{W}_{u}$ (in yellow), associated to a given $\mathcal{S}_{u}^{*}$ subset (in black) of the full terminal set $\mathcal{S}^{*}$ (in red). The codimension-two set $\mathcal{V}_{u}$ is the (possibly disconnected) boundary of Cauchy surfaces $\Sigma_{u}$ for $\mathcal{W}_{u}$.

fundamental. These two constructions are not completely equivalent, since the WdW patch anchored at $\mathcal{V}_{u}$ may fail to intersect $\mathcal{S}^{*}$ at sufficiently 'early times'. In this paper we are more interested in the asymptotic 'late-time' behavior in which $\mathcal{W}_{u}$ does have a nontrivial boundary component at the singularity. Therefore, we tacitly adopt in what follows the nesting construction of the WdW patches and we will often refer to the associated complexity measures as 'nesting complexity'.

In the $\mathrm{AC} / \mathrm{VC}$ heuristic correspondence, the codimension-two surfaces $\mathcal{V}_{u}$ are the natural analogs of the transition surface with volume of order $S$ in figure 1 . Therefore, interpreting the $u$ coordinate as a (null) time variable, we are led to the following definition of nesting complexity associated to the given family of $\mathrm{WdW}$ patches $\mathcal{W}_{u}$,

$$
C_{u}^{*}=\alpha I\left[\mathcal{W}_{u}\right]+\frac{\lambda}{4 G} \operatorname{Area}\left[\mathcal{V}_{u}\right]
$$

where $\alpha$ is a normalization factor, $\lambda$ is an undetermined constant which sets the relative importance of the codimension-two boundary counterterm, and $I\left[\mathcal{W}_{u}\right]$ denotes the on-shell gravitational action, now integrated over the WdW patch $\mathcal{W}_{u}$. The action can be written as $I=I_{\text {bulk }}+I_{\partial}$, separating bulk and boundary contributions. The bulk term has the standard form

$$
I\left[\mathcal{W}_{u}\right]_{\text {bulk }}=\frac{1}{16 \pi G} \int_{\mathcal{W}_{u}}\left(R-2 \Lambda+\mathcal{L}_{m}\right)+\ldots
$$


where the dots stand for higher-derivative corrections and $\mathcal{L}_{m}$ is the Lagrangian density for non-gravitational degrees of freedom, out of which we have explicitly singled out the cosmological constant.

The boundary term $I_{\partial}$ requires special consideration. On general grounds, it is given by a sum of contributions from codimension-one and codimension-two components of the boundary $\partial \mathcal{W}_{u}$. The non-null codimension-one pieces and their joints are given by the standard York-Gibbons-Hawking (YGH) term and a set of well understood joint contributions (see [21] for a review). On the other hand, some formal choices are necessary in the presence of null codimension-one pieces, and the physics behind these choices remains somewhat unclear (see for example the considerations in $[19,21,22]$ ).

For the purposes of this paper, we make a minimal choice for $I_{\partial}$ in which we only retain the YGH term for non-null codimension-one components and we drop the contributions from codimension-one null components and their codimension-two joints. We do this while keeping open the possibility that a further understanding of the microscopic definition of complexity will require the specification of non-geometrical quantities in the AC rules.

Applying these rules to the WdW patches (2.4) we have an action

$$
I\left[\mathcal{W}_{u}\right]=I\left[\mathcal{W}_{u}\right]_{\text {bulk }}+I\left[\mathcal{S}_{u}^{*}\right]_{\mathrm{YGH}}
$$

where the bulk term is given by (2.6) and the YGH term is restricted to the spacelike singular component of the WdW patch,

$$
I\left[\mathcal{S}_{u}^{*}\right]_{\mathrm{YGH}}=\frac{1}{8 \pi G} \int_{\mathcal{S}_{u}^{*}} K .
$$

Hence, the unpacked ansatz for the nesting complexity reads

$$
C_{u}^{*}=\alpha I\left[\mathcal{W}_{u}\right]_{\text {bulk }}+\alpha I\left[\mathcal{S}_{u}^{*}\right]_{\mathrm{YGH}}+\frac{\lambda}{4 G} \operatorname{Area}\left[\mathcal{V}_{u}\right]
$$

Although we have chosen to regard the entropy counterterm as separate from $I_{\partial}$, we may as well consider it as one more boundary contribution to the action. ${ }^{3}$ In this case we are effectively picking out boundary components supported on $\mathcal{S}_{u}^{*} \cup \mathcal{V}_{u}$, rather than the full $\partial \mathcal{W}_{u}$. This instruction admits a nice topological interpretation, namely it amounts to focusing on the intersection of the boundaries of $J^{-}\left(\mathcal{S}_{u}^{*}\right)$ and $D^{-}\left(\mathcal{S}^{*}\right)$, rather than the boundary of the intersection:

$$
\mathcal{S}_{u}^{*} \cup \mathcal{V}_{u}=\partial J^{-}\left(\mathcal{S}_{u}^{*}\right) \cap \partial D^{-}\left(\mathcal{S}^{*}\right),
$$

a relation that may be used to provide an invariant definition of $\mathcal{V}_{u}$ given the family of nested sets $\mathcal{S}_{u}^{*}$.

Once this nesting complexity is defined, we can now recover the notion of 'total complexity flow' into the singularity, which was loosely defined in (2.2), as the asymptotic limit of the nesting procedure. More precisely, we have

$$
C\left[\mathcal{S}^{*}\right]=\lim _{u \rightarrow u_{*}} C_{u}^{*},
$$

\footnotetext{
${ }^{3}$ Incidentally, this would correspond to a very special case of the prescription introduced in [21], in which one drops the codimiension-one null pieces and adjusts the normalization conventions of affine parameters in an ad hoc, $u$-dependent manner.
} 


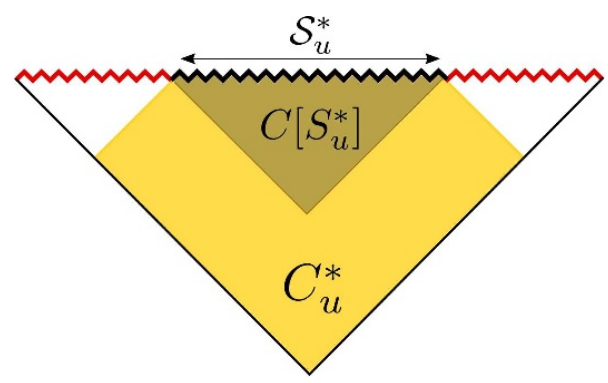

Figure 6. The difference between $C_{u}^{*}$ and $C\left[\mathcal{S}_{u}^{*}\right]$ as determined by the different domains of integration.

It is important to notice that, when considering singular subsets $\mathcal{S}_{u}^{*}$, the nesting complexity $C_{u}^{*}$ is different from the 'total complexity' $C\left[\mathcal{S}_{u}^{*}\right]$ flowing into $\mathcal{S}_{u}^{*}$, as shown in figure 6 . In other words, we regard $C\left[\mathcal{S}^{*}\right]$ as the AC-analog of $\mathcal{S}_{\infty}$, (cf. figure 3 ), and $C_{u}^{*}$ as the ACanalog of $\mathcal{S}_{t}^{\prime}$, (cf. figure 4).

In the rest of the paper we shall see that the definition of $C_{u}^{*}$ given in (2.9) satisfies two interesting properties of a general character. The first is the existence of a local component defined at space-like singularities which can be reached by a coarse-graining procedure (section 3), and the second is the conjectured monotonicity of the nesting complexity $C_{u}^{*}$, to be discussed in section 4 below.

\section{The local component of the terminal complexity}

A remarkable property of the $\mathrm{AC}$ complexity prescription is the occurrence of a quantitatively important contribution coming directly from the singularity, through the evaluation of the YGH term. Since this is a term in the low-energy effective action, we should be suspicious of its validity. On the other hand, we are instructed to take this contribution seriously down to its precise dependence on coefficients, as this is crucial for the claimed uniformity of the growth law (2.1) for AdS black holes in various dimensions, large and small. In a similar vein, the contribution (or lack of it) of the YGH term at the singularities is crucial for the 'non-computing' behavior in various systems, such as AdS black holes in the $1 / d$ expansion [20] and cold hyperbolic black holes [18, 19].

These considerations suggest that complexity is actually the piece of the holographic dictionary which most efficiently 'sees' the properties of the singularities.

The YGH contribution is local and formally extensive over the singular set $\mathcal{S}^{*}$. However, the volume form is not generally defined at $\mathcal{S}^{*}$, which makes the notion of 'extensivity' non-trivial. In order to elucidate this point, let us parametrize the near-terminal metric by a Gaussian normal coordinate $\tau$. This foliates the near-terminal spacetime into spacelike surfaces $\Sigma_{\tau}$, according to the proper-time distance to $\mathcal{S}^{*}$. In defining a metric on the $\Sigma_{\tau}$ slices, we extract a conventional power of the proper time according to the ansatz

$$
d s^{2}=-d \tau^{2}+(\tau H)^{2 \gamma / d} d \Sigma_{\tau}^{2}
$$


Here, $H$ is an inverse-length setting a characteristic value for the expansion away from the terminal set. In general, the $d$-dimensional metric $d \Sigma_{\tau}^{2}$ does not have a smooth limit as $\tau \rightarrow 0^{+}$, but we may choose the conventional exponent $\gamma$ in such a way that its volume form does have a smooth limit. We shall actually assume that this volume form is analytic in $\tau$, since this will be a property of all examples we study (it would be interesting to assess the generality of this assumption). We will refer to such notion of volume for $\mathcal{S}^{*}$ as the 'comoving volume' of the terminal set and denote its measure as $d \mathrm{Vol}_{c}$.

In this notation, the YGH term in the action is computed as

$$
I\left[\mathcal{S}^{*}\right]_{\mathrm{YGH}}=\frac{1}{8 \pi G} \lim _{\tau \rightarrow 0^{+}} \partial_{\tau}\left[(H \tau)^{\gamma} \operatorname{Vol}_{c}\left[\Sigma_{\tau}\right]\right] .
$$

Picking the term proportional to the comoving volume $\operatorname{Vol}_{c}\left[\mathcal{S}^{*}\right]$ of the singular set, we find that the YGH term vanishes for $\gamma>1$ and is infinite for $\gamma<1$, except perhaps the case $\gamma=0$ where the answer depends on the possible occurrence of logarithmic terms in the terminal expansion near $\tau=0$. The most interesting case is $\gamma=1$, for which one defines a nontrivial 'comoving complexity density' at the singular set, given by $H / 8 \pi G$.

The black hole singularity has $\gamma=1$ and thus presents a purely local contribution to complexity. In fact, this feature appears to be quite general. At spherically symmetric black-hole singularities we have a vanishing $\mathbf{S}^{d-1}$ and an expanding 'radial' direction. Hence, the metric is locally of the Kasner form, i.e.

$$
d s^{2}=-d \tau^{2}+\sum_{j=1}^{d}(H \tau)^{2 p_{j}} d \sigma_{j}^{2},
$$

with a particular choice of Kasner parameters $p_{c}=2 / d$ for $d-1$ 'crunching' directions and $p_{r}=-1+2 / d$ for the 'ripping' direction. More generally, the Kasner parameters are restricted to satisfy the sum rules $\sum_{j} p_{j}=\sum_{j} p_{j}^{2}=1$ and any such metric can be put in the form (3.1) with $\gamma=1$, with 'comoving' metric

$$
d \Sigma_{\tau}^{2}=\sum_{j}(\tau H)^{2 p_{j}-2 / d} d \sigma_{j}^{2} .
$$

In particular, it has a smooth comoving volume form,

$$
d \operatorname{Vol}_{c}\left[\Sigma_{\tau}\right]=\wedge_{j=1}^{d} d \sigma_{j}
$$

as a simple consequence of the sum rule $\sum_{j} p_{j}=1$.

The $\gamma=1$ property and the resulting non-vanishing 'complexity density' persist if we let the Kasner parameters depend smoothly on the 'longitudinal' $\sigma_{j}$. In fact, the classic results of refs. [7-9] (BKL) indicate that such a 'generalized Kasner' metrics furnish a good local approximation of the near-singular region (after a slight generalization involving local rescalings and frame rotations).

\subsection{Evanescent terminal complexity}

If we regard the generalized Kasner behavior as 'generic' we may say that spacelike singularities tend to have a non-vanishing local complexity density. On the other hand, there 
are important examples of singularities whose YGH contribution vanishes, such as those occurring in standard FRW spacetimes.

To bring this simple point home, we can apply (3.2) to the standard FRW metric

$$
d s^{2}=-d \tau^{2}+a(\tau)^{2} d \Sigma^{2},
$$

with a singularity at $\tau=0$. By construction, the non-singular comoving volume is just given by the volume of the homogeneous and isotropic surfaces $\Sigma$, so that the complexity exponent $\gamma$ can be read off from the short-time asymptotics of the scale factor $a(\tau)$. Since FRW metrics require non-trivial matter degrees of freedom, we follow standard practice and model them as a perfect fluid with squared speed of sound equal to $w=p / \rho$, where $p$ denotes the pressure and $\rho$ the energy density. Then, we have the standard solution $\rho a^{d(1+w)}=$ constant, which leads to $a(\tau) \sim \tau^{2 / d(1+w)}$ or, equivalently

$$
\gamma_{\mathrm{FRW}}=\frac{2}{1+w} .
$$

This result implies that the only FRW singularity with a finite complexity density is the slightly unphysical case with 'stiff matter', $w=1$, leading to $\gamma=1$. On the other hand, a formally infinite contribution to the complexity density, associated to $\gamma<1$, would require $w>1$ in the FRW context, i.e. a violation of the physical conditions on the matter degrees of freedom.

Imposing the physical condition that the matter is strictly below the 'stiff' limit, $w<1$, we have $\gamma>1$, implying a vanishing local complexity. Hence, we find that 'ordered' singularities of FRW type have a vanishing local contribution to holographic complexity, just as it happened with the Weyl criterion of Penrose.

It turns out that there is an interesting twist to this story. According to the classic BKL analysis [7-9], the vicinity of a generic spacelike singularity is not quite described by a single generalized Kasner metric, but rather an oscillating regime where a series of 'epochs' succeed one another, each epoch being locally described by a generalized Kasner solution of the type (3.3). The values of the Kasner parameters, $p_{j}$, change from one epoch to the next in a deterministic but chaotic manner. In addition, the frame determining the special coordinates $\sigma_{j}$ in (3.3) undergoes a rotation, and furthermore the induced volume form at fixed $\tau$ is rescaled by a finite factor which we may absorb in the dimensionful expansion parameter $H$. Hence, in the $n$-th epoch we have a metric

$$
\left.d s^{2}\right|_{(n)}=-d \tau^{2}+\left(H_{n} \tau\right)^{2 / d} d^{2} \Sigma_{\tau}^{(n)},
$$

where $d^{2} \Sigma_{\tau}^{(n)}$ is a rotated version of (3.4) with Kasner parameters $p_{j}^{(n)}$. All epochs are described by $\gamma=1$ metrics but, crucially, they have slightly different parameters $p_{j}^{(n)}$ and $H_{n}$. In particular, the substitution rule for the expansion parameter is

$$
H_{n+1}=\left(2 p_{r}^{(n)}+1\right) H_{n}
$$

where $p_{r}^{(n)}<0$ is the 'ripping' parameter of the $n$-th epoch. Since $2 p_{r}^{(n)}+1<1$ for all $n$, the series of $H_{n}$ is monotonically decreasing. 
If we compute the YGH contribution to complexity by placing a regulating surface and taking the limit, the result of the complexity density is determined by the limit of $H_{n}$. Namely it is proportional to

$$
\prod_{\text {epochs }}\left(2 p_{r}+1\right)
$$

According to the analysis of [7-9] the truly generic singularity features an infinite number of Kasner epochs. In this situation the product (3.10), featuring an infinite set of numbers in the open interval $(0,1)$, is bound to vanish for almost all singularities. We refer to this phenomenon as the 'evanescence' of the local complexity for a generic BKL singularity. We may argue that, ultimately, a cutoff at Planck time from the singularity must be imposed but, in any case, the complexity computed by this ansatz would have a suppression factor determined by the number of epochs taking place until Planck time. These arguments suggest that the generic singularity is not that different from the FRW one, and the standard black-hole singularities are the 'special ones' regarding complexity.

\subsection{Local terminal complexity and coarse-graining}

The remarkable properties of the local YGH contribution beg the question of whether we may be able to isolate this term in more physical terms. A natural strategy in this case is to focus on the extensivity of the local contribution, a property not shared by the full $\mathrm{AC}$ complexity. We can illustrate this point by focusing on the simpler case of vacuum solutions.

A vacuum solution is a $(d+1)$-dimensional Einstein manifold whose metric satisfies

$$
R_{\mu \nu}=\frac{2 \Lambda}{d-1} g_{\mu \nu}
$$

with cosmological constant $\Lambda$ and no matter degrees of freedom. The bulk contribution to the on-shell action is then proportional to the spacetime volume

$$
I[X]_{\mathrm{bulk}}=\frac{1}{16 \pi G} \int_{X}(R-2 \Lambda)=\frac{\Lambda}{4 \pi G(d-1)} \operatorname{Vol}[X] .
$$

Assuming a $\gamma=1$ singular set $\mathcal{S}^{*}$ with non-vanishing complexity density, we have a full complexity given formally by

$$
C\left[\mathcal{S}^{*}\right]=\alpha \frac{\Lambda}{4 \pi G(d-1)} \operatorname{Vol}\left[D^{-}\left(\mathcal{S}^{*}\right)\right]+\alpha \frac{H}{8 \pi G} \operatorname{Vol}_{c}\left[\mathcal{S}^{*}\right]
$$

While the YGH term is extensive along the comoving volume of $\mathcal{S}^{*}$, the bulk contribution is extensive in the full spacetime volume of the past domain of dependence. Considering the case $\Lambda<0$, as corresponds to states in an AdS/CFT context, we have a negative-definite bulk contribution, leading to a 'subextensivity' property of the full quasilocal complexity. Indeed, under a coarse-graining of the singular set $\mathcal{S}^{*}=\cup_{i} \mathcal{S}_{i}^{*}$ as indicated in figure 7 , the expression (3.13) satisfies

$$
C\left[\mathcal{S}^{*}\right]=C\left[\cup_{i} \mathcal{S}_{i}^{*}\right] \leq \sum_{i} C\left[\mathcal{S}_{i}^{*}\right]
$$




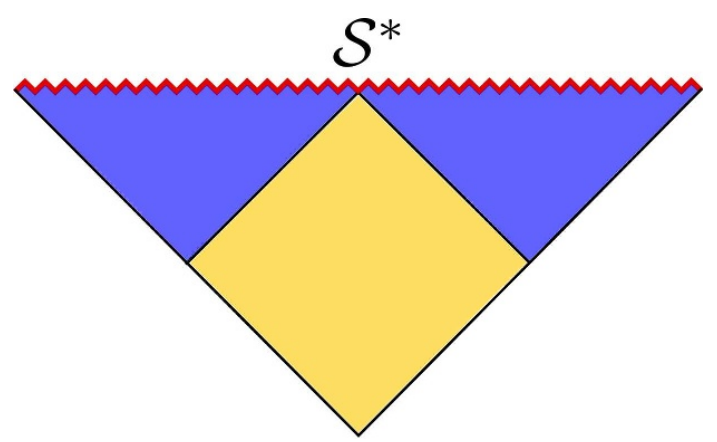

Figure 7. When the bulk action is dominated by a negative cosmological constant, the bulk contribution to the AC terminal complexity of $\mathcal{S}^{*}$ is subextensive. While the YGH contribution is extensive over $\mathcal{S}^{*}$, the bulk contribution is more negative for the larger set (yellow) than it is for the union of the smaller sets (blue).

The inequality is reversed (corresponding to superextensivity) for vacuum singularities in $\Lambda>0$ spaces. The deviation from extensivity would disappear if the bulk contributions were to become negligible, a situation we may expect in the limit of extreme coarse graining, illustrated in figure 8.

In this case, the limit of infinite coarse-graining does isolate the YGH term. To be more precise, we require that the bulk contributions be consistently smaller than the YGH contribution for small subsets of $\mathcal{S}^{*}$. We can check this explicitly for $\gamma=1$ vacuum singularities described by (3.13) and admitting a local Kasner description. Let us consider a fine partition of the singular set by subsets $\mathcal{S}_{\epsilon}^{*}$ with comoving volume of order $\epsilon^{d}$. The condition for the bulk contribution to be negligible for small sets is that

$$
\frac{\operatorname{Vol}\left[D^{-}\left(\mathcal{S}_{\epsilon}^{*}\right)\right]}{\operatorname{Vol}_{c}\left[\mathcal{S}_{\epsilon}^{*}\right]} \sim \epsilon^{a}
$$

with $a>0$. Instead of computing the volume of the past domain of dependence, $D^{-}\left(\mathcal{S}_{\epsilon}^{*}\right)$, it is easier to compute the volume of the larger set $B^{-}\left(\mathcal{S}_{\epsilon}^{*}\right)$, which 'boxes' it in the standard coordinate frame. If $\tau_{\epsilon}$ is the maximal value of the $\tau$ coordinate in $D^{-}\left(\mathcal{S}_{\epsilon}^{*}\right)$, the $\epsilon$-box is defined by the full $\tau \leq \tau_{\epsilon}$ subset with given comoving coordinates covering $\mathcal{S}^{*}$, cf. figure 9. Evidently, $\operatorname{Vol}\left[B^{-}\left(\mathcal{S}_{\epsilon}^{*}\right)\right] \geq \operatorname{Vol}\left[D^{-}\left(\mathcal{S}_{\epsilon}^{*}\right)\right]$, so that it is enough to establish the condition $(3.15)$ for $B^{-}\left(\mathcal{S}_{\epsilon}^{*}\right)$.

In order to construct explicitly $B^{-}\left(\mathcal{S}_{\epsilon}^{*}\right)$ for the Kasner metric $(3.3)$ we define $\mathcal{S}_{\epsilon}^{*}$ to be a $d$-dimensional cube in the $\sigma$ coordinates with common extent $\Delta \sigma_{j}=\epsilon$. Its comoving volume is $\operatorname{Vol}_{c}\left[\mathcal{S}_{\epsilon}^{*}\right]=\epsilon^{d}$ and the past domain of dependence, $D^{-}\left(\mathcal{S}_{\epsilon}^{*}\right)$, is a trapezoid with base $\mathcal{S}_{\epsilon}^{*}$ and a ridge with the topology of a $(d-1)$-dimensional cube, determined by the intersection of light rays in the spacetime plane with faster past-convergence. For any direction $\sigma_{j}$ we can define a corresponding conformal time coordinate $\eta_{j}$ such that light rays propagate with unit slope in the $\left(\eta_{j}, \sigma_{j}\right)$ plane. The explicit relation between $\eta_{j}$ and the proper time is

$$
\left(1-p_{j}\right) H \eta_{j}=(H \tau)^{1-p_{j}}
$$




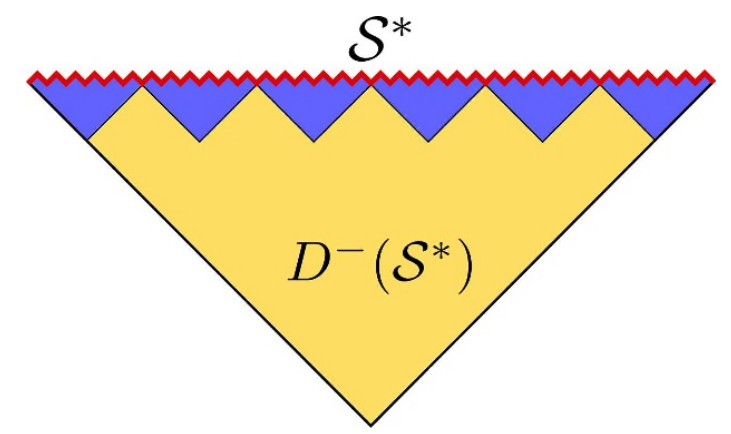

Figure 8. When the bulk volume remains sufficiently bounded in the vicinity of the terminal surface, the local complexity of $\mathcal{S}^{*}$ results from the limit of an extreme coarse graining.

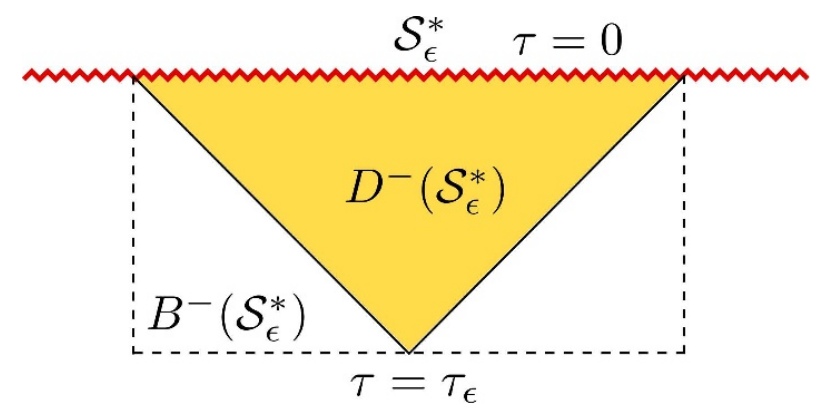

Figure 9. Comparison between the $\epsilon$-box and the past domain of dependence of $\mathcal{S}_{\epsilon}^{*}$.

where $p_{j}$ is the Kasner exponent in the direction $\sigma_{j}$. Light rays whose $\sigma_{j}$ separation is $\epsilon$ at $\tau=0$ converge in the past at $\tau_{\epsilon}^{(j)}$ given by

$$
H \tau_{\epsilon}^{(j)}=\left(\left(1-p_{j}\right) \frac{H \epsilon}{2}\right)^{\frac{1}{1-p_{j}}} .
$$

Thus, the past domain of dependence of the full $\mathcal{S}_{\epsilon}^{*}$ set is determined by the smallest $\tau_{\epsilon}^{(j)}$ or, equivalently, by the largest Kasner exponent which we denote by $p_{c}$ :

$$
H \tau_{\epsilon}=\left(\left(1-p_{c}\right) \frac{H \epsilon}{2}\right)^{\frac{1}{1-p_{c}}} .
$$

With these ingredients we can compute the volume of the $\epsilon$-box as

$$
\mathrm{Vol}\left[B^{-}\left(\mathcal{S}_{\epsilon}^{*}\right)\right]=\int_{0}^{\tau_{\epsilon}} d \tau(H \tau) \epsilon^{d}=\frac{1}{2 H}\left(\tau_{\epsilon} H\right)^{2} \epsilon^{d},
$$

and verify $(3.15)$ with $a=2\left(1-p_{c}\right)^{-1}$.

For solutions with matter degrees of freedom we need to check that the Lagrangian $\mathcal{L}_{m}$ is not too singular. For instance, if we have a FRW terminal with metric

$$
d s^{2}=-d \tau^{2}+a(\tau)^{2} d \Sigma^{2}
$$

and squared speed of sound $w=(2-\gamma) / \gamma$, the energy density scales as

$$
\rho \sim a(\tau)^{-d(1+w)} \sim \tau^{-2} .
$$


Approximating the action dimensionally as the volume integral of the energy density, we estimate

$$
I\left[B^{-}\left(\mathcal{S}_{\epsilon}^{*}\right)\right]_{\mathrm{bulk}} \propto \epsilon^{d} \int_{0}^{\tau_{\epsilon}} d \tau \tau^{\gamma} \frac{1}{\tau^{2}} \sim \epsilon^{d} \tau_{\epsilon}^{\gamma-1} .
$$

The condition for the coarse-graining procedure to be well-defined is now

$$
\frac{I\left[B^{-}\left(\mathcal{S}_{\epsilon}^{*}\right)\right]_{\text {bulk }}}{\operatorname{Vol}_{c}\left[\mathcal{S}_{\epsilon}^{*}\right]} \sim \epsilon^{a}, \quad a>0 .
$$

The physical condition that the matter equation of state remains strictly below the stiff limit, $w<1$, implies that $\gamma>1$ and thus (3.23) is satisfied provided $\tau_{\epsilon}$ scales with a positive power of $\epsilon$. This happens for any solution which decelerates away from the singularity, since the FRW conformal time is given by

$$
H \eta=\frac{d}{d-\gamma}(H \tau)^{\frac{d-\gamma}{d}} .
$$

It is precisely for decelerating singularities that we have $\gamma<d$ and $\tau_{\epsilon} \sim \epsilon^{\frac{d}{d-\gamma}}$ scaling with a positive power of $\epsilon$, leading to an automatically well-defined coarse-graining limit.

The situation is less clear for FRW metrics that accelerate away from the singularity, corresponding to $\gamma \geq d$. Now the FRW conformal time plummets to $-\infty$ as $\tau \rightarrow 0^{+}$. The problem in this case is that $D^{-}\left(\mathcal{S}_{\epsilon}^{*}\right)$ is not itself well defined, as any past light cone emanating from $\tau=0$ and converging at a finite value $\tau_{0}$ subtends an infinite comoving volume at the terminal surface. To address this point we regularize the terminal surface by bringing it slightly before the singularity at $\tau=\delta$, as indicated in figure (10). In other words, we compute the past domain of dependence for a small, $\epsilon$-sized subset of $\Sigma_{\delta}$ rather than $\mathcal{S}^{*}$. Let us denote this set $\mathcal{S}_{\epsilon}^{\delta}$. Its past domain of dependence, $D^{-}\left(\mathcal{S}_{\epsilon}^{\delta}\right)$, has an earliest proper time which is a function of both $\epsilon$ and $\delta$,

$$
\tau_{0}(\epsilon, \delta)=\frac{1}{H}\left((H \delta)^{\frac{d-\gamma}{d}}+\frac{d-\gamma}{d} \frac{H \epsilon}{2}\right)^{\frac{d}{d-\gamma}} .
$$

For $\gamma>d$, this quantity vanishes linearly in $\delta$ as the terminal time cutoff is removed at fixed $\epsilon$. Hence, when we repeat the estimate (3.22) we find that

$$
I\left[B^{-}\left(\mathcal{S}_{\epsilon}^{\delta}\right)\right]_{\mathrm{bulk}} \propto \epsilon^{d} \int_{\delta}^{\tau_{0}} d \tau \tau^{\gamma} \frac{1}{\tau^{2}} \sim \epsilon^{d}\left(\tau_{0}^{\gamma-1}-\delta^{\gamma-1}\right) \longrightarrow 0
$$

as $\delta \rightarrow 0$ at fixed $\epsilon$, since both terms vanish in the limit. Therefore, the bulk contribution vanishes when we remove the regularization at fixed comoving volume, even before we take $\epsilon \rightarrow 0$.

The borderline case of a fluid with a stiff matter equation of state, i.e. $\gamma=1$, requires a separate analysis. From (3.22) we can see that the bulk action diverges logarithmically near the singularity, so that a regularization procedure will be needed as well. Following the same notation as in figure 10, we get

$$
\frac{I\left[B^{-}\left(\mathcal{S}_{\epsilon}^{\delta}\right)\right]_{\mathrm{bulk}}}{\operatorname{Vol}_{c}\left[\mathcal{S}_{\epsilon}^{\delta}\right]} \sim \log \left(\frac{\tau_{0}(\epsilon, \delta)}{\delta}\right),
$$




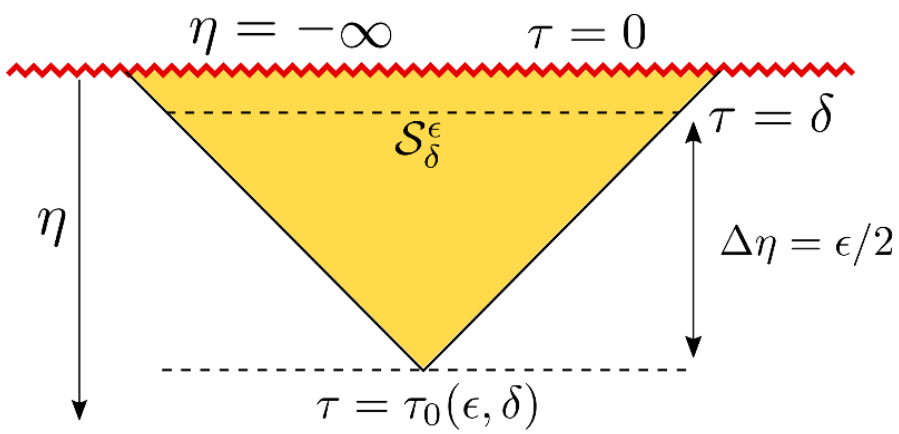

Figure 10. The regularized terminal $\mathcal{S}_{\epsilon}^{\delta}$ and its past domain of dependence.

whose behavior as $\epsilon, \delta \rightarrow 0$ does depend on the order of the limits. For fixed regularization parameter $\delta$ we have the expansion

$$
\frac{\tau_{0}(\epsilon, \delta)}{\delta} \sim 1+\mathcal{O}(\epsilon)
$$

which makes (3.27) approach linearly to zero as $\epsilon \rightarrow 0$. On the other hand, if we try to remove the regularization at fixed $\epsilon$ we find

$$
\frac{\tau_{0}(\epsilon, \delta)}{\delta} \sim \frac{1}{H \delta}(H \epsilon)^{\frac{d}{d-1}}+\mathcal{O}\left(\delta^{-\frac{1}{d}}\right)
$$

which diverges as $\delta \rightarrow 0$. Hence, the FRW singularity with stiff matter does not have a consistent coarse-graining limit which would isolate the local complexity density. ${ }^{4}$

On the other hand, the more 'physical' FRW singularities with $\gamma>1$ are extremely 'thin' in any measure of local complexity. For $1<\gamma<d$, corresponding to metrics that decelerate away from the singularity, we have a vanishing local contribution from the YGH term and a well-defined coarse-graining procedure isolating this term. For $\gamma \geq d$, the situation is even more extreme, since the quasilocal complexity vanishes for sets of small but fixed comoving volume.

We interpret these features as holographic analogs of the Weyl curvature criterion by Penrose, which also gave a smallest measure of complexity to FRW cosmologies. The main difference here is that the holographic notion of complexity is rather more refined, making a quantitative distinction between accelerating and decelerating cosmologies.

\section{Terminal monotonicity}

In this section we return to the full quasilocal complexity and discuss the monotonicity of its 'nesting' properties. Given that our definition of $C_{u}^{*}$ in (2.4) was tailored to mimic the role of the cut surface $\mathcal{S}_{t}^{\prime}$ in the $\mathrm{VC}$ ansatz, we expect that $C_{u}^{*}$ should increase monotonically, at least asymptotically as $u \rightarrow u_{*}$. Since this may depend on the choice of 'entropic' coupling $\lambda$, we conjecture that an appropriate choice exists such that this always happens.

\footnotetext{
${ }^{4}$ The consistency of the coarse-graining procedure would need to be analyzed anew if extra boundary terms are included in the action, beyond our minimal choice in (2.9) and (2.11).
} 
In other words, we conjecture that the full on-shell action of the WdW patch $I\left[\mathcal{W}_{u}\right]$ is either monotonically increasing as $u \rightarrow u^{*}$ or, in case it decreases, it does so at a rate bounded (in absolute magnitude) by that of Area $\left[\mathcal{V}_{u}\right]$.

A priori, it is not immediately obvious that such monotonicity will hold. Let us consider again vacuum solutions satisfying (3.11). Given a set of WdW patches $\mathcal{W}_{u}$ associated to a family of singular subsets $\mathcal{S}_{u}^{*}$ with $\gamma=1$, and holding codimension-two 'edges' $\mathcal{V}_{u}$, we can write the full 'nesting' complexity as a function of $u$ as arising from three contributions:

$$
\left.C_{u}^{*}\right|_{\mathrm{VAC}}=\alpha \frac{\Lambda}{4 \pi G(d-1)} \operatorname{Vol}\left[\mathcal{W}_{u}\right]+\alpha \frac{H}{8 \pi G} \operatorname{Vol}_{c}\left[\mathcal{S}_{u}^{*}\right]+\frac{\lambda}{4 G} \operatorname{Area}\left[\mathcal{V}_{u}\right]
$$

The first term comes from the bulk Einstein-Hilbert action and is extensive in space-time volume of the WdW patch. The second term is the terminal density, extensive in the comoving volume of the terminal set, and the last term is the 'entropic' counterterm.

A glance at the expression (4.1) indicates that the monotonicity is not obvious, in particular for singularities embedded in AdS, since there one finds $\Lambda<0$ and the bulk contribution is negative as the space-time volume of the $\mathrm{WdW}$ patches grows. In addition, the particular monotonicity properties of Area $\left[\mathcal{V}_{u}\right]$ could affect the final answer. On the other hand, the space-time volume vanishes near singularities of Einstein's equations. Thus, we expect the main contribution to the on-shell action to come from the 'corners' of the WdW patch, i.e. the vicinity of the sets $\mathcal{V}_{u}$. In such a situation we may expect that any threat to monotonicity coming from the bulk action could eventually be fixed by an appropriate choice of the entropic coupling $\lambda$.

Short of a general proof, we have examined a number of examples in which the explicit computation can be carried reliably and found agreement with the monotonicity conjecture. We consider here a few extreme cases which illustrate the qualitatively different roles played by the entropic counterterm proportional to $\lambda$ in (2.5). Our choices are motivated by the ability to compute exactly the complexity on terminal WdW patches using the specific formula (4.1), but also by our interest in exposing as much as possible the contrast between entropy and complexity when referred to cosmological singularities. Quite generally, we can associate an entropic measure to a terminal set $\mathcal{S}^{*}$ by the limit of the codimension-two areas Area $\left[\mathcal{V}_{u}\right]$ in Planck units, as $u \rightarrow u_{*}$, i.e.

$$
S\left[\mathcal{S}^{*}\right] \equiv \lim _{u \rightarrow u_{*}} \frac{\operatorname{Area}\left[\mathcal{V}_{u}\right]}{4 G} .
$$

With this definition, the full singularity of a standard black hole solution has finite entropy, whereas any proper portion of the singularity has zero entropy. On the other hand, there are prototypical cosmological singularities with infinite entropy, such as the interior of a Coleman-de Luccia bubble in vacuum decay. It is very interesting to keep track of the terminal complexity in these wildly different situations from the point of view of the entropy as a measure of the 'holographic dimensionality' of the relevant state spaces.

Before embarking in our tour of examples, we would like to comment briefly on the relation to previous work. In [1] the VC complexity was estimated for a number of cosmological singularities which are naturally embedded into concrete AdS/CFT constructions. 
In these examples it was found that a regularized version of the VC complexity was monotonically decreasing on approaching the singularity, in contrast with our statement here for the cuasilocal complexity. A similar behavior was obtained for the AC ansatz in the same examples by [2]. The reason for this apparent discrepancy is simply that the full complexity computed in [1] is dominated by UV contributions to the VC ansatz, and these are highly dependent on the particular details of the embedding into asymptotically AdS geometries. For instance, some of the examples are based on singular CFT metrics which shrink to zero size, and others involve expanding domain walls in the bulk. In the first case it is natural that the UV contribution to complexity should have a negative derivative in time, as corresponds to a shrinking Hilbert space on the full CFT. In the second case, a time-dependent conversion between UV and IR degrees of freedom is introduced in the CFT by switching on a relevant operator with a time-dependent coupling, and the c-theorem explains why the UV again dominates the balance. Therefore, there is no contradiction since the two monotonicity statements refer to different quantities. The positive monotonicity of the quasilocal complexity defined here (by restriction of the AC/VC ansatz to the interior of $D^{-}\left(\mathcal{S}^{*}\right)$ ), is compatible with the negative monotonicity of the full complexity, particularly when the latter is dominated by a strong UV time-dependence.

Vacuum terminals with constant entropy. We begin our tour of examples with the benchmark case of a (future) black hole interior. The standard case is provided by the spherical AdS black hole solution, with an $\mathbb{R} \times \mathrm{SO}(d)$ isometry group and metric

$$
d s^{2}=-f(r) d t^{2}+\frac{d r^{2}}{f(r)}+r^{2} d \Omega_{d-1}^{2}, \quad f(r)=1+\frac{r^{2}}{\ell^{2}}-\frac{\mu}{r^{d-2}},
$$

where $\ell$ is the curvature radius of AdS. The vicinity of the singularity at $r=0$ is controlled by a single length scale, $\mu^{\frac{1}{d-2}}$, which relates to the horizon radius $R$ through

$$
\mu=R^{d-2}+\frac{R^{d}}{\ell^{2}}
$$

The near-terminal metric can be written in the form (3.1) with $\gamma=1$ and

$$
H=\left(\frac{2}{d \cdot \mu}\right)^{\frac{1}{d-2}}
$$

The terminal set at $r=0$ has topology $\mathbf{R} \times \mathbf{S}^{d-1}$, and its comoving metric degenerates through a stretching of the $\mathbf{R}$ factor and a contraction of the sphere. We can parametrize the terminal set by the homogeneous $t$-coordinate along the $\mathbf{R}$ factor. Let $\mathcal{S}_{\Delta t}^{*}$ denote a subset $\mathbf{I}_{\Delta t} \times \mathbf{S}^{d-1}$ of the terminal set, where $\mathbf{I}_{\Delta t} \subset \mathbf{R}$ is an interval of length $\Delta t$ along the $t$ coordinate. Its comoving volume is then given by

$$
\operatorname{Vol}_{c}\left[\mathcal{S}_{\Delta t}^{*}\right]=\Delta t \Omega_{d-1} H^{1-d},
$$

where $\Omega_{d-1}=\operatorname{Vol}\left[\mathbf{S}^{d-1}\right]$. The YGH contribution to the on-shell action is

$$
I\left[\mathcal{S}_{\Delta t}^{*}\right]_{\mathrm{YGH}}=\frac{H}{8 \pi G} \operatorname{Vol}_{c}\left[\mathcal{S}_{\Delta t}^{*}\right]=\frac{d \mu \Omega_{d-1} \Delta t}{16 \pi G},
$$




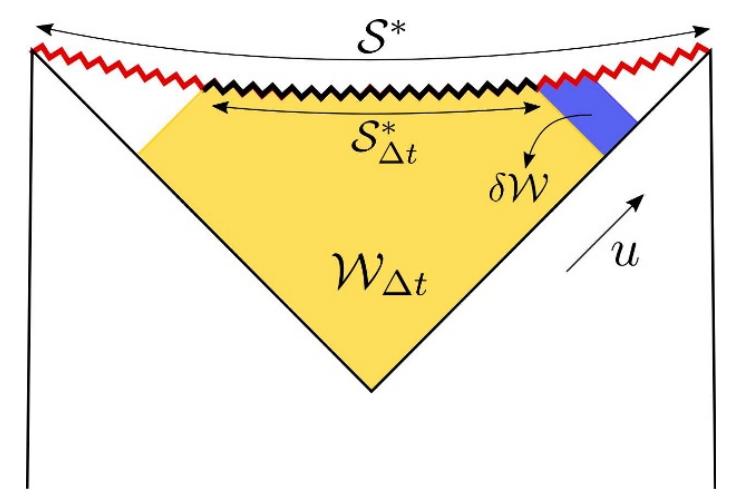

Figure 11. The terminal WdW patch $\mathcal{W}_{\Delta t}$ within the black-hole interior and the small stripe $\delta \mathcal{W}$.

for both small and large black holes. Expressed in terms of physical quantities, we find a contribution to complexity which is positive and proportional to $\Delta t M$, where $M$ is the mass of the black hole.

The bulk contribution (3.12) is negative-definite. In order to compute it, we follow [21] and introduce an infalling Eddington-Finkelstein coordinate

$$
u=t+\int^{r} \frac{d r^{\prime}}{f\left(r^{\prime}\right)}
$$

to write the metric in the form

$$
d s^{2}=-f(r) d u^{2}+2 d u d r+r^{2} d \Omega_{d-1}^{2}
$$

This allows us to compute the integral (3.12) as

$$
I\left[W_{\Delta t}\right]_{\text {bulk }}=-\frac{\Omega_{d-1} d}{8 \pi G \ell^{2}} \int_{\mathcal{W}_{\Delta t}} d u d r r^{d-1}
$$

To show monotonicity, it suffices to evaluate the action over a stripe of $u$-extent $\delta u$, as indicated in figure 11, and we obtain

$$
I[\delta \mathcal{W}]_{\text {bulk }}=-\frac{\Omega_{d-1} d}{8 \pi G \ell^{2}} \int_{\delta u} d u \int_{0}^{R} d r r^{d-1}=-\frac{\Omega_{d-1} R^{d}}{8 \pi G \ell^{2}} \delta u
$$

Adding the contribution from the YGH term and using the relation $\mu=R^{d-2}+R^{d} / \ell^{2}$, we find that

$$
\delta I=\delta u \frac{\Omega_{d-1} R^{d-2}}{8 \pi G \ell^{2}}\left(d \ell^{2}+(d-1) R^{2}\right)
$$

is always positive, implying the positive monotonicity of $C_{\Delta t}^{*}$. In establishing this result, the 'entropic' counterterm proportional to $\lambda$ plays no role, since the horizon area is asymptotically constant. 
Vacuum terminals with divergent entropy. A qualitatively different situation is obtained for terminals that look like standard cosmologies of FRW type. In general, such FRW models require non-trivial contributions from matter degrees of freedom. In order to stay within the realm of vacuum solutions, so that we can still apply (4.1), we look at FRW metrics arising in the interior of vacuum bubbles of Coleman-de Luccia type. A crucial property of any such bubble is that it expands, asymptotically approaching the speed of light, so that the area of bounding codimension-two surfaces is guaranteed to diverge, i.e. they always have divergent entropy.

As an explicit example with an exact solution we can consider a kind of 'topological crunch' spacetime (see for example [1,23-25]). In this construction, we have a sort of higher-dimensional generalization of the BTZ black hole, i.e. the metric is locally pure $\mathrm{AdS}_{d+2}$, but a convenient identification by the group of integers realizes a time-dependent compactification with topology $\mathbf{S}^{1} \times \operatorname{AdS}_{d+1}$, where the $\mathbf{S}^{1}$ fiber shrinks to zero size at the singular locus. More precisely, we consider the following metric on $D^{-}\left(\mathcal{S}^{*}\right)$,

$$
d s^{2}=-d t^{2}+\ell^{2} \sin ^{2}(t / \ell) d \mathbf{H}_{d}^{2}+\ell^{2} \cos ^{2}(t / \ell) d \phi^{2},
$$

where $\phi$ is an angle parametrizing the additional compact circle, $d \mathbf{H}_{d}^{2}$ is the standard unit metric on the $d$-dimensional Euclidean hyperboloid and $\ell$ is the AdS radius of curvature. The singularity at $t=\ell \pi / 2$, occurring when the compact circle degenerates to vanishing size, is very mild, but enough to guarantee that the terminal set $\mathcal{S}^{*}$ of topology $\mathbf{S}^{1} \times \mathbf{H}_{d}$ has zero physical volume. The comoving volume of $\mathcal{S}^{*}$ is given by the standard volume forms on $\mathbf{S}^{1} \times \mathbf{H}_{d}$ times $\ell^{d+1}$.

This model has the nice feature of admitting a straightforward embedding into AdS/CFT. On the exterior of $D^{-}\left(\mathcal{S}^{*}\right)$ we can realize the $\mathrm{SO}(1, d)$ isometry on timelike de Sitter hypersurfaces instead of spacelike hyperbolic ones. Hence, the model can be embedded as a de Sitter-invariant state of a $\mathrm{CFT}_{d+1}$ defined on $\mathrm{dS}_{d} \times \mathbf{S}^{1}$.

In order to perform the required computations, it is useful to define null coordinates on $D^{-}\left(\mathcal{S}^{*}\right)$ as follows. First, we introduce a radial coordinate $\chi$ over $\mathbf{H}_{d}$ and a conformal time variable $\eta=2 \tan ^{-1}\left(e^{t / \ell}\right)$ over the $\operatorname{AdS}_{d+1}$ factor, so that the metric is written in the form

$$
d s^{2}=\ell^{2} \sin ^{2}(t / \ell)\left[-d \eta^{2}+d \chi^{2}+\sinh ^{2}(\chi) d \Omega_{d-1}^{2}\right]+\ell^{2} \cos ^{2}(t / \ell) d \phi^{2} .
$$

Next, we introduce null coordinates

$$
\tan U=e^{\eta+\chi}, \quad \tan V=e^{\eta-\chi},
$$

and write the complete metric as

$$
d s^{2}=\ell^{2} \sec ^{2}(U-V)\left[-4 d U d V+\sin ^{2}(U-V) d \Omega_{d-1}^{2}+\cos ^{2}(U+V) d \phi^{2}\right] .
$$

We consider a set of nested $(d+2)$-dimensional WdW patches $\mathcal{W}_{u}$ bounded by the coordinate $u$, where $u=U-\pi / 4 \geq 0$. The bulk action is then given by

$$
I\left[\mathcal{W}_{u}\right]_{\text {bulk }}=-\frac{(d+1) \Omega_{d-1} \ell^{d}}{2 G} \int_{\mathcal{W}(U, V)} d U d V \frac{\tan ^{d-1}(U-V)}{\cos ^{3}(U-V)} \cos (U+V),
$$




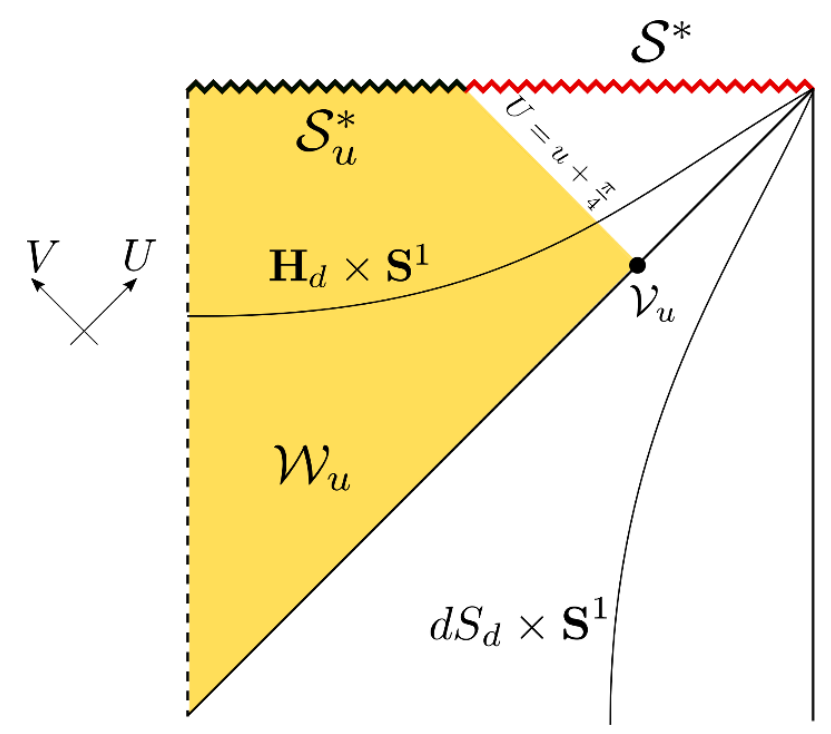

Figure 12. The causal structure of the topological crunch singularity and its associated WdW patches. The projection of $\mathcal{W}_{u}$ onto the $(U, V)$ plane is shown as the yellow sector in the figure, and coincides with the domain of integration $\mathcal{W}(U, V)$.

where the domain of integration $\mathcal{W}(U, V)$ in the $(U, V)$ plane is shown in the figure 12 . Notice that Newton's constant $G$ has now the appropriate dimensionality for a $(d+2)$ dimensional spacetime, explaining the power of $\ell$ in the numerator. A rather explicit expression can be obtained for the variation with respect to the $u$ coordinate,

$$
\frac{d}{d u} I\left[\mathcal{W}_{u}\right]_{\text {bulk }}=-\frac{\Omega_{d-1} \ell^{d}}{2 G d}\left[(d-\sin (2 u))(\tan (u+\pi / 4))^{d}+\sin (2 u)(\tan (2 u))^{d}\right],
$$

and the behavior near the singularity at $u_{*}=\pi / 4$ is

$$
\frac{d}{d u} I\left[\mathcal{W}_{u}\right]_{\mathrm{bulk}} \approx-\frac{\Omega_{d-1} \ell^{d}}{2 G d}\left(d+2^{-d}-1\right) \frac{1}{\left(u_{*}-u\right)^{d}} .
$$

A similarly explicit expression can be obtained for the variation of the YGH term:

$$
\frac{d}{d u} I\left[\mathcal{S}_{u}^{*}\right]_{\mathrm{YGH}}=\frac{\Omega_{d-1} \ell^{d}}{2 G} \frac{(\tan (2 u))^{d}}{\sin (2 u)} \approx \frac{\Omega_{d-1} \ell^{d}}{2 G} 2^{-d} \frac{1}{\left(u_{*}-u\right)^{d}},
$$

where the last expression is the asymptotic limit near the singularity. We find that, for all $d \geq 2$, the sum of (4.19) and (4.20) is negative, approaching minus-infinity as $u \rightarrow u_{*}$. On the other hand, one finds that the entropic term has the same degree of divergence near the singularity:

$$
\text { Area }\left[\mathcal{V}_{u}\right]=2 \pi \Omega_{d-1} \ell^{d}(\tan (u+\pi / 4))^{d-1},
$$

and the rate near the singuarity is given by

$$
\frac{d}{d u} \operatorname{Area}\left[\mathcal{V}_{u}\right] \approx 2 \pi \Omega_{d-1} \ell^{d} \frac{d-1}{\left(u_{*}-u\right)^{d}}
$$


Hence, the terminal complexity of the topological crunch has the expected monotonicity property provided we choose

$$
\frac{\lambda}{\alpha}>\frac{1}{\pi d}\left(1-2^{-d}\right)
$$

This example differs from the eternal black hole in that the 'entropy of the singularity' grows indefinitely, as measured by $\operatorname{Area}\left(\mathcal{V}_{u}\right)$, and the associated coupling $\lambda$ must satisfy a lower bound in order to guarantee monotonicity of $C_{u}^{*}$.

An even simpler model of this kind is obtained by removing the $\mathbf{S}^{1}$ factor in (4.13). The FRW patch of pure $\mathrm{AdS}_{d+1}$ has a coordinate singularity at $t=\pi \ell$, but we may render it a true singularity by considering a thin-walled bubble with $\mathrm{dS}_{d}$ wordvolume hitting the boundary of $\mathrm{AdS}_{d+1}$ right at the boundary of the $t=\pi \ell$ null surface (cf. figure 13). This model represents an approximate de Sitter-invariant condensate state for a CFT on $\mathrm{dS}_{d}$ (cf. $[1,26,27]$ ).

Using the same null coordinates introduced in (4.15), we have a metric

$$
d s^{2}=\ell^{2} \sec ^{2}(U-V)\left[-4 d U d V+\sin ^{2}(U-V) d \Omega_{d-1}^{2}\right],
$$

The singularity sits at $U=\pi / 2$ in the null limit. We can now consider the set of nested WdW patches $\mathcal{W}_{u}$ defined by $0 \leq U \leq u$ and $V \leq U$. These patches do not actually touch the singularity in the idealized null limit, as shown in figure 13, so that the monotonicity properties of $C_{u}^{*}$ depend entirely on the balance between the negative-definite bulk contribution and the volume of the codimension-two boundary sets $\mathcal{V}_{u}$. The bulk action is given by

$$
I\left[\mathcal{W}_{u}\right]_{\text {bulk }}=-\frac{d \ell^{d-1} \Omega_{d-1}}{8 \pi G} \int_{0}^{u} d U \int_{0}^{U} d V \frac{\tan ^{d-1}(U-V)}{\cos ^{2}(U-V)} \approx-\frac{\ell^{d-1} \Omega_{d-1}}{8 \pi G(d-1)}\left(\frac{1}{\frac{\pi}{2}-u}\right)^{d-1}
$$

where the last expression is an approximation for $u \approx \pi / 2$. On the other hand, the volume of the codimension-two set is

$$
\text { Area }\left[\mathcal{V}_{u}\right]=\ell^{d-1} \Omega_{d-1} \tan ^{d-1}(u) \approx \ell^{d-1} \Omega_{d-1}\left(\frac{1}{\frac{\pi}{2}-u}\right)^{d-1}
$$

We thus conclude that monotonicity is guaranteed provided we pick an entropy coupling $\lambda$ satisfying the inequality

$$
\frac{\lambda}{\alpha}>\frac{1}{2 \pi(d-1)}
$$

In a sense, the singularity induced by a vacuum bubble in the thin-wall limit provides the extreme case in which the monotonicity is threatened by a negative, diverging, bulk contribution. Here we see that this negative infinity is always tamed by a sufficiently large, but ultimately finite, entropic counterterm. It would be interesting to obtain these results in more realistic constructions going beyond the thin-wall approximation by the inclusion of explicit dynamical scalar fields. 


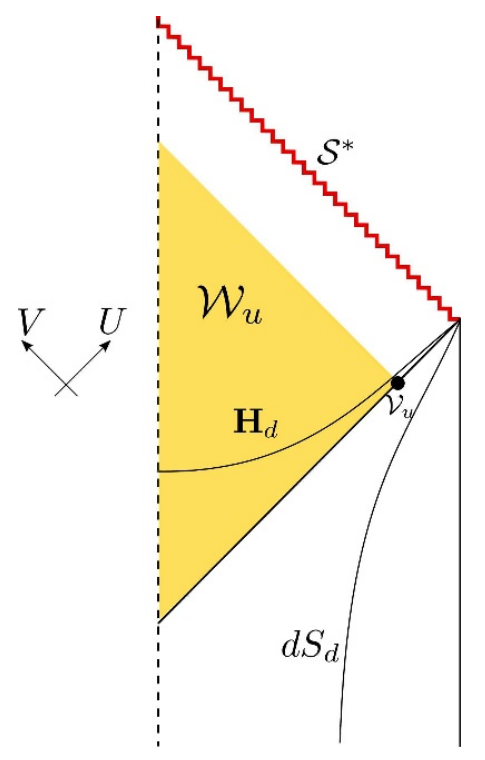

Figure 13. The idealized state generating a null singularity by the collision of a thin-walled bubble with $\mathrm{dS}_{d}$ worldvolume.

A vacuum terminal with vanishing entropy. In this section we consider an example in which the entropy of the singularity, as defined by the volume of codimension-two sets $\mathcal{V}_{u}$, has precisely the opposite behavior to the topological crunch model, namely it vanishes at the singular locus. Consider the Kasner metric in $d+1$ dimensions

$$
d s^{2}=-d t^{2}+\sum_{i=1}^{d}(t H)^{2 p_{i}} d x_{i}^{2},
$$

the standard vacuum solution with zero cosmological constant and $\mathbb{R}^{d}$ symmetry and, as discussed at length in the previous section, a local approximation for 'small portions' of more general singularities (cf. [7-10]). We recall that the coefficients $p_{i}$ are restricted to satisfy $\sum_{i} p_{i}=\sum_{i} p_{i}^{2}=1$, and at least one of the exponents $p_{i}$ must be negative, indicating that at least one direction stretches as one approaches the singularity. The volume of codimension-two surfaces sitting at some constant value of $t$ always vanishes as $|t|$ in the $t \rightarrow 0$ limit.

For simplicity of computations, we shall consider a family of sets $\mathcal{S}_{u}^{*}$ adapted to the symmetry of the metric, defined as follows: in the $\mathbf{R}^{d}$ parametrized by the coordinates $x_{j}$, we single out one particular direction, $x_{u}$, and pick an interval $\mathbf{I}_{u}=\left[-\frac{u}{2}, \frac{u}{2}\right]$ of length $u$ in this direction. The set of nested terminal sets is then defined as the 'slabs' $\mathcal{S}_{u}^{*}=\mathbf{R}^{d-1} \times \mathbf{I}_{u}$. The full terminal set is obtained by taking the limit $u \rightarrow u_{*}$ with some finite $u_{*}$. The causal structure of the WdW patches is shown in figure 14. The codimension-two surfaces $\mathcal{V}_{u}$ controlling the entropic coupling sit on the surfaces $t=t(u)$, with $t(u)$ determined by the equation

$$
\left(1-p_{u}\right)\left(u_{*}-u\right)=4 t(u)^{1-p_{u}},
$$

and $p_{u}$ is the Kasner exponent along the compact direction of the slab $\mathcal{S}_{u}^{*}$. 


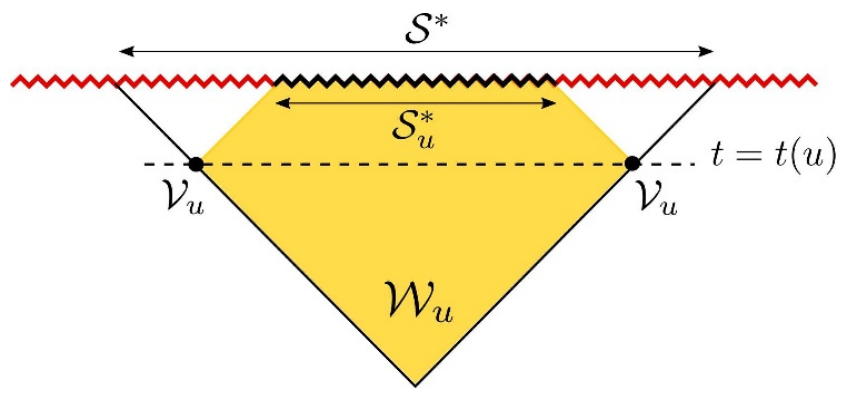

Figure 14. WdW patch for a Kasner slab $\mathcal{S}_{u}^{*}=\left[-\frac{u}{2}, \frac{u}{2}\right] \times \mathbf{R}^{d-1}$.

Since (4.28) has already the form (3.1) with $\gamma=1$, we obtain an extensive and positive contribution from the YGH term

$$
I\left[\mathcal{S}_{u}^{*}\right]_{\mathrm{YGH}}=\frac{H}{8 \pi G} \operatorname{Vol}\left[\mathbf{R}^{d-1}\right] u,
$$

where we interpret the volume of $\mathbf{R}^{d-1}$ in the sense of defining the appropriate density along the non-compact directions. Moreover, the Ricci-flatness property of (4.28) implies that there is no bulk contribution to the on-shell action, and we seem to obtain a monotonic result for $C_{u}^{*}$ with no explicit reference to the entropic coupling $\lambda$. On the other hand, a simple computation shows that

$$
\operatorname{Area}\left[\mathcal{V}_{u}\right]=\left(1-p_{u}\right) H \operatorname{Vol}\left[\mathbf{R}^{d-1}\right] \frac{u_{*}-u}{2},
$$

which vanishes as $u \rightarrow u_{*}$, giving a monotonically decreasing contribution to complexity as soon as we have $\lambda>0$. Therefore, in order to ensure the right monotonicity property of $C_{u}^{*}$ we must assume that the entropic coupling satisfies an upper bound

$$
\frac{\lambda}{\alpha}<\frac{1}{\pi\left(1-p_{u}\right)}
$$

independently of whether the slab's finite interval is laid along a ripping $\left(p_{u}<0\right)$ or crunching $\left(p_{u}>0\right)$ direction.

Unlike the Coleman-de Luccia bubble, the Kasner situation has a finite complexity in the asymptotic limit $u \rightarrow u_{*}$, but the entropy counterterm could make the total complexity approach the limit from above rather than from below. If we want to ensure a positive rate in this case we must prevent the entropic coupling from being too large, as indicated in the bound (4.32).

\section{Conclusions and outlook}

In this paper we have introduced quasilocal notions of AC complexity for terminal sets such as spacelike singularities in General Relativity. The basic idea is to build WdW patches restricted to the past causal domain of the singular set. Holographic data are associated to codimension-two surfaces on corresponding horizons. In principle, we can assign a notion of entropy to a singularity by looking at the area of these codimension-two sets. 
One of the main observations made in this note is that the on-shell action of the WdW patches should be defined as monotonically increasing under the nesting of WdW patches, independently of the behavior of the entropy. We have tested this idea on a number of exactly solvable examples comprising the three qualitative behaviors with respect to the entropy: asymptotically constant entropy like in black-hole interiors, diverging entropy as in the interior of Coleman-de Luccia bubbles, and vanishing entropy as in the Kasner spacetime. In all cases one can define monotonic terminal $\mathrm{AC}$ complexities at the price of adjusting an additive contribution proportional to the entropy. It would be interesting to back these checks with actual proofs, under the assumption of appropriate energy conditions.

The YGH term evaluated at the singularity plays a special role. First, we emphasize that it is to be taken seriously despite the fact that it is a contribution to the low-energy effective action extrapolated beyond its natural realm of applicability. Second, we have shown that one may isolate it as the local contribution to the complexity under an extreme coarse-graining procedure. In this respect, the relation of the YGH term to the full quasilocal complexity is analogous to the relation between the classical thermodynamic entropy, obtained through coarse-graining, and the exact von Neumann entropy of a quantum many-body state.

Another interesting property of the local complexity is its vague resemblance to Penrose's Weyl curvature criterion. In particular, FRW singularities are singled out by having vanishing complexity density, according to this definition. The similarity is not perfect though, since one can identify various differences. First, the 'lack of complexity' seems to be even more severe for FRW metrics which accelerate away from the singularity. Second, within the local description of generic singularities, as presented in the classic BKL analysis, our ansatz assigns a vanishing complexity density to the formal infinite sequences of chaotic Kasner 'epochs'. Since these chaotic structures are generic in the light of the BKL analysis, we would conclude that the local complexity density of generic spacelike singularities is zero. In this sense, complexity would behave similarly to local gravitational energy in General Relativity: while global and quasilocal definitions of gravitational energy exist, any attempt at a fully local definition is doomed to failure because of the equivalence principle. We find the parallel with complexity unveiled here quite intriguing.

A question of potential interest is the generalization of these concepts to asymptotically de Sitter spacetimes. This is natural since the asymptotic future of de Sitter is also a 'causal terminal' and one may consider the behavior of the quantities defined in this paper. In fact, a simple check shows that every term in the quasilocal complexity ansatz becomes infinite in this case. Both the YGH term evaluated at the asymptotic future and the bulk action diverge. It turns out that in this case the YGH term dominates and formally gives an infinitely negative complexity. It would be interesting to elucidate these questions further.

\section{Acknowledgments}

We would like to thank M. Alishahiha, B. Freivogel, C. Gomez, J. Maldacena, R. Myers, E. Rabinovici, J. Martinez-Magan and K. Sousa for discussions on various aspects of compu- 
tational complexity, as well as the participants of the 'It for Qbit Workshop' at Bariloche and 'Complexity Workshop 2018' at AEI-Postdam, where preliminary versions of this work were presented. This work is partially supported by the Spanish Research Agency (Agencia Estatal de Investigación) through the grants IFT Centro de Excelencia Severo Ochoa SEV-2016-0597 and FPA2015-65480-P. The work of J.M.G. is funded by Fundación La Caixa under "La Caixa-Severo Ochoa" international predoctoral grant.

Open Access. This article is distributed under the terms of the Creative Commons Attribution License (CC-BY 4.0), which permits any use, distribution and reproduction in any medium, provided the original author(s) and source are credited.

\section{References}

[1] J.L.F. Barbón and E. Rabinovici, Holographic complexity and spacetime singularities, JHEP 01 (2016) 084 [arXiv:1509.09291] [INSPIRE].

[2] S. Bolognesi, E. Rabinovici and S.R. Roy, On Some Universal Features of the Holographic Quantum Complexity of Bulk Singularities, JHEP 06 (2018) 016 [arXiv:1802.02045] [INSPIRE].

[3] L. Susskind, Entanglement is not enough, Fortsch. Phys. 64 (2016) 49 [arXiv:1411.0690] [INSPIRE].

[4] A.R. Brown, D.A. Roberts, L. Susskind, B. Swingle and Y. Zhao, Holographic Complexity Equals Bulk Action?, Phys. Rev. Lett. 116 (2016) 191301 [arXiv:1509.07876] [INSPIRE].

[5] A.R. Brown, D.A. Roberts, L. Susskind, B. Swingle and Y. Zhao, Complexity, action and black holes, Phys. Rev. D 93 (2016) 086006 [arXiv:1512.04993] [InSPIRE].

[6] C.W. Misner, Mixmaster universe, Phys. Rev. Lett. 22 (1969) 1071 [InSPIRE].

[7] V.A. Belinsky, I.M. Khalatnikov and E.M. Lifshitz, Oscillatory approach to a singular point in the relativistic cosmology, Adv. Phys. 19 (1970) 525.

[8] V.A. Belinsky, I.M. Khalatnikov and E.M. Lifshitz, A General Solution of the Einstein Equations with a Time Singularity, Adv. Phys. 31 (1982) 639.

[9] V.A. Belinskii, E.M. Lifshitz and I.M. Khalatnikov, 30 Years of the Landau Institute Selected Papers. "Construction of a General Cosmological Solution of the Einstein Equation with a Time Singularity", World Scientific, New York U.S.A. (1996), pg. 763.

[10] V. Belinski and M. Henneaux, The Cosmological Singularity, Cambridge University Press, Cambridge U.K. (2017).

[11] R. Penrose, Singularities and time-asymmetry, in General Relativity: An Einstein Centenary Survey, Akademie-Verlag, Berlin Germany (1979), pg. 581.

[12] S. Ryu and T. Takayanagi, Holographic derivation of entanglement entropy from AdS/CFT, Phys. Rev. Lett. 96 (2006) 181602 [hep-th/0603001] [INSPIRE].

[13] V.E. Hubeny, M. Rangamani and T. Takayanagi, A Covariant holographic entanglement entropy proposal, JHEP 07 (2007) 062 [arXiv:0705.0016] [INSPIRE].

[14] M. Alishahiha, Holographic Complexity, Phys. Rev. D 92 (2015) 126009 [arXiv:1509.06614] [INSPIRE]. 
[15] S. Lloyd, Ultimate physical limits to computation, Nature 406 (2000) 1047 [quant-ph/9908043].

[16] W. Cottrell and M. Montero, Complexity is simple!, JHEP 02 (2018) 039 [arXiv: 1710.01175] [INSPIRE].

[17] D. Carmi, S. Chapman, H. Marrochio, R.C. Myers and S. Sugishita, On the Time Dependence of Holographic Complexity, JHEP 11 (2017) 188 [arXiv:1709.10184] [INSPIRE].

[18] J.L.F. Barbón and J. Martín-García, Holographic Complexity Of Cold Hyperbolic Black Holes, JHEP 11 (2015) 181 [arXiv:1510.00349] [INSPIRE].

[19] S. Chapman, H. Marrochio and R.C. Myers, Complexity of Formation in Holography, JHEP 01 (2017) 062 [arXiv: 1610.08063] [INSPIRE].

[20] J.L.F. Barbón and J. Martín-García, Holographic non-computers, JHEP 02 (2018) 181 [arXiv: 1710.06415] [INSPIRE].

[21] L. Lehner, R.C. Myers, E. Poisson and R.D. Sorkin, Gravitational action with null boundaries, Phys. Rev. D 94 (2016) 084046 [arXiv:1609.00207] [InSPIRE].

[22] A. Reynolds and S.F. Ross, Divergences in Holographic Complexity, Class. Quant. Grav. 34 (2017) 105004 [arXiv:1612.05439] [INSPIRE].

[23] M. Bañados, A. Gomberoff and C. Martinez, Anti-de Sitter space and black holes, Class. Quant. Grav. 15 (1998) 3575 [hep-th/9805087] [INSPIRE].

[24] M. Bañados, Constant curvature black holes, Phys. Rev. D 57 (1998) 1068 [gr-qc/9703040] [INSPIRE].

[25] J. Maldacena and G.L. Pimentel, Entanglement entropy in de Sitter space, JHEP 02 (2013) 038 [arXiv:1210.7244] [INSPIRE].

[26] J.L.F. Barbon and E. Rabinovici, AdS Crunches, CFT Falls And Cosmological Complementarity, JHEP 04 (2011) 044 [arXiv: 1102.3015] [INSPIRE].

[27] J.L.F. Barbón and E. Rabinovici, Conformal Complementarity Maps, JHEP 12 (2013) 023 [arXiv: 1308.1921] [INSPIRE]. 